(C) 2015 IEEE. Personal use of this material is permitted. Permission from IEEE must be obtained for all other uses, in any current or future media, including reprinting/republishing this material for advertising or promotional purposes, creating new collective works, for resale or redistribution to servers or lists, or reuse of any copyrighted component of this work in other works. 


\section{Bi-Decomposition using Boolean Relations}

\author{
Anna Bernasconi \\ Dipartimento di Informatica \\ Università di Pisa, Italy \\ anna.bernasconi@unipi.it
}

Gabriella Trucco

Dipartimento di Informatica

Università degli Studi di Milano, Italy

gabriella.trucco@unimi.it

\author{
Robert K. Brayton \\ Department of EECS \\ University of California, Berkeley, USA \\ brayton@berkeley.edu
}

\author{
Valentina Ciriani \\ Dipartimento di Informatica \\ Università degli Studi di Milano, Italy \\ valentina.ciriani@unimi.it
}

\author{
Tiziano Villa \\ Dipartimento di Informatica \\ Università degli Studi di Verona, Italy \\ tiziano.villa@univr.it
}

\begin{abstract}
We study three-level implementations where the first two levels represent a standard PLA form with an ANDplane and an OR-plane. This implements a $2 m$-output SOP. The final stage consists of $m$ two-input programmable LUTs. The PLA outputs are paired so that the LUT outputs implement a set of $m$ given incompletely specified functions (ISFs). Three-level structures have been studied previously, e.g. resulting in ANDOR-AND or AND-OR-XOR implementations. By using the LUT effectively, the composition of the AND-plane can be controlled to implement a PLA which has the optimum phase assignment for maximum cube sharing. For each output, we characterize the problem of all legal implementations of such a model, by defining Boolean relations that capture all the flexibility induced by the final LUT logic. The extra LUT level provides a dimension beyond simple phase assignment. We performed experiments using a Boolean relation minimizer to compare such realizations vs. SOP forms and published three-level forms, comparing areas and delays. To approximate the possible sharing in the PLA, we mapped the $2 m$ PLA logic using SIS. We focused on experiments with two-input Boolean functions not captured by AND-OR-AND or AND-OR-XOR approaches and found good gains in many cases with affordable increases in synthesis runtimes.
\end{abstract}

\section{INTRODUCTION}

Functional decomposition rewrites a logic function $f(X)$ as the composition of a set of functions $h, g_{1}, g_{2}, \ldots, g_{m}$ such that $f(X)=h\left(g_{1}(X), g_{2}(X), \ldots, g_{m}(X)\right)([16]$, [22]). It is a fundamental tool in logic synthesis for multi-level and FPGA implementations, and in the theory of circuit and communication complexity. The simplest case when $m=2$, called bidecomposition, appears often in the literature, because it fits quite well with the usual representations of logic as networks of 2-input gates, and if applied recursively generates more general decompositions ([21], [14]). Since the decomposition is top down and performed at the output level for each primary output separately, by bi-decomposition we transform initial two-level forms into three-level forms, about which there is an extensive literature for comparison. Afterwards, the remaining logic may be handled by standard synthesis methods, like twolevel minimization of the two blocks followed by technology mapping onto a given implementation library.

In this work we study three-level forms, aiming at implementing incompletely specified functions (ISFs), $f=$ $\left(f_{o n}, f_{o f f}\right)$, using a cover $c=u$ op $v$, where op is a two input gate or LUT, and $u$ and $v$ are two SOP forms. For an example with $o p(u, v)=\bar{u} v$, see the scheme depicted in Figure 2 .

For an ISF $f$ represented by its on-set $f_{o n}$, dc-set $f_{d c}$, and off-set $f_{o f f}$, a cover $g$ is a completely specified function such that $f_{o n} \subseteq g \subseteq f_{o n} \cup f_{d c}$. A cover $h$ of its complement ISF, $f_{\text {off }} \subseteq h \subseteq f_{\text {off }} \cup f_{d c}$, may lead to a more optimal implementation.

Example: Consider op $(u, v)=\bar{u}+v=(u \Rightarrow v)$. We have the flexibility to implement a minterm in $f_{o n}$ in one of three ways: by adding it to both the cover $v$ and the cover $u$ (the output of the latter is negated and becomes a 0 input to the $O R$ gate), or by adding it to the cover $v$ and by not adding it to the cover $u$ (i.e., it is put in $\bar{u}$ so that the 0 output of $u$ is negated and becomes a 1 input to the $O R$ gate), or by not adding it to the cover $v$ and not adding it to the cover $u$ (whose negated output inputs a 1 into the $O R$ gate). In other words, a point in the onset can be realized by $v$ only, or by $v$ and $\bar{u}$, or by $\bar{u}$ only.

Intuitively, a LUT (or gate) at the output provides a generalization of the problem of choosing the best output phase assignment in the realization of a $2 m$ SOP (see [17], [18]). With a LUT at the output, we can choose the best phase assignment for the SOP feeding the LUT where the SOP has $2 m$ outputs. Even if the $2 m$ outputs were implemented as a Boolean network, there is still an interesting phase assignment because we can choose a cover of the ISF $f$ or a cover of its complement ISF. The example also illustrates the case where a two-input operator connecting two logic blocks $u$ and $v$ induces don't care conditions: e.g., when $o p=O R$, if a point in the on-set is added to $v$ then we do not care if it is added also to $u$, yielding the don't care condition $(-1)$ for the two outputs $u$ and $v$; dually, the same outputs can assume values in the cube $(1-)$, since if an on-set point is added to $u$ we do not care if it is part of $v$. However, the two cubes $(1-)$ and $(-1)$ cannot be expressed by a single cube (which would mean that it could be expressed as a don't care), but instead a Boolean relation is required to model this flexibility. Therefore, the problem of optimizing the implementation of a decomposition of $f$ in the form $c=u$ op $v$, is one of defining and optimizing a Boolean relation, depending on $o p$. In this paper, we experiment with a set of ops which have one of its inputs inverted. We compare our implementations of such complemented-input circuits synthesized by a Boolean relation minimizer, BREL 
[1], against published results that use special minimizers for deriving AND-OR-AND, OR-AND-OR, and AND-OR-XOR three-level forms. The use of a Boolean relation minimizer on such problems is not new [1] but experiments and comparisons in this context have not been done.

The paper is organized as follows: we show a motivating example in Sec. II, we summarize briefly previous work in Sec. III, Boolean relations in Sec. IV, and describe in Sec. V the Boolean relations characterizing completely the flexibility in the realization of op circuits. In Sec. VI, we report experimental results comparing our forms against SOPs (with different phase assignments) and specialized three-level minimizers like AOXMIN [10], after running SIS to compute areas and delays of the underlying SOPs; the experiments show average gains of around $20-30 \%$ in the majority of benchmarks. In Sec. VII we draw conclusions and discuss possible future research.

\section{Motivating ExAmple}

In order to better describe the proposed approach we give here a simple example of the bi-decomposition $f_{0} \Rightarrow f_{1} \equiv$ $\bar{f}_{0}+f_{1}$ for the function $f$ depicted in Figure 1(a). We first recall that, by the De Morgan laws, a complemented SOP (Sum of Products) can be seen as a POS (Product of Sums) form with the same number of literals. For example: $\overline{x_{1} \bar{x}_{2}+x_{1} x_{3} \bar{x}_{4}}=$ $\left(\bar{x}_{1}+x_{2}\right)\left(\bar{x}_{1}+\bar{x}_{3}+x_{4}\right)$.

Considering the function $f$ represented by the Karnaugh map in Figure 1(a). If we compute a standard SOP cover we have the minimal SOP in Figure 1(b):

$$
f_{S O P}=f_{1}^{S O P}=x_{1} \bar{x}_{2}+x_{1} x_{3}+x_{1} x_{4}+\bar{x}_{2} x_{3}+\bar{x}_{2} x_{4},
$$

that has 10 literals. The corresponding minimal POS form in Figure 1(c) is:

$$
f_{P O S}=\bar{f}_{0}^{P O S}=\left(x_{1}+\bar{x}_{2}\right)\left(x_{1}+x_{3}+x_{4}\right)\left(\bar{x}_{2}+x_{3}+x_{4}\right),
$$

containing 8 literals. In this case it is convenient to represent the function as the negation of its offset with 3 products and 8 literals, against a cost of 5 products and 10 literals if we represent its onset. Moreover, we can do even better if we enlarge the offset to include the onset point 1000, because we save 1 product and 4 literals in the representation of the offset; however, we must represent the onset point 1000 by adding a product of the onset that covers it, paying a penalty of 1 product and 2 literals, with an overall cost of 3 products and 6 literals (better than 3 products and 8 literals). In conclusion, a minimal $f_{0} \Rightarrow f_{1}$ circuit for $f$ in Figure $1(\mathrm{~d})$ is:

$$
f_{B}=\bar{f}_{0}+f_{1}=\left(\left(x_{1}+\bar{x}_{2}\right)\left(x_{3}+x_{4}\right)\right)+x_{1} \bar{x}_{2}
$$

that contains 6 literals (it is $f_{0}=\bar{x}_{1} x_{2}+\bar{x}_{3} \bar{x}_{4}$ and $f_{1}=x_{1} \bar{x}_{2}$ ). Note that in the last Karnaugh map (Figure 1(d)) the point 1000 is in the the OFF set of $\bar{f}_{0}$ but is in the ON set of $f_{1}$, thus is in the ON set of the OR between $\bar{f}_{0}$ and $f_{1}\left(\bar{f}_{0}+f_{1}\right)$. Moreover, the points 1001,1010 , and 1011 are covered by both $\bar{f}_{0}$ and $f_{1}$. We can conclude that it is useful to define a strategy that finds the best cover $f_{B}=\bar{f}_{0}+f_{1}$. Note that, in general, the best solution could be $f_{B}=f_{1}$ (i.e., SOP) or $f_{B}=\bar{f}_{0}$ (i.e., POS or complemented SOP).

\section{PREVIOUS WORK}

Three-level logic has been studied for decades, a reason being that three levels are enough to produce a minimal network for most Boolean functions (see Sasao, [19]). The minimization of various forms of three-level logic has been studied in the literature, e.g., AND-OR-AND networks consisting of two SOPs with a two-input AND gate at the output (Malik, [13] and Dubrova, [9]); OR-AND-OR networks (see Sasao, [20], and Debnath, [8]); AND-OR-EXOR networks, called EX-SOP, with a single two-input EXOR gate at the output (see Debnath, [6], [7] and Dubrova, [10]); EXORAND-OR networks, called SPPs (Sums of Pseudo-Products) which generalize SOP expressions by replacing products of literals with products of EXOR gates (see Luccio, [12]), further restricted to k-SPPs where EXOR factors contain at most $k$ literals and to 2-SPPs (see Ciriani, [4], [5]) for which an efficient ESPRESSO-like minimization procedure has been designed (see Bernasconi, [2]).

A way to obtain three-level forms is to apply one step of bi-decomposition, which decomposes a given logic function $F(X)$ into three blocks as $F(X)=G(X)$ op $H(X)$, where $o p$ is a two-input gate (usually AND, OR, or EXOR) (see Sasao, [21] and Mishchenko, [14]). A strong bi-decomposition has the form $G\left(X_{1}, X_{3}\right)$ op $H\left(X_{3}, X_{2}\right)$ where $X_{1}, X_{2}, X_{3}$ is a partition of the input variables; When $X_{2}=\emptyset$ the bidecomposition is weak. In this paper we address the special case of weak decompositions where $X_{1}=X_{2}=\emptyset$. Some interesting results on bi-decomposition are described in [11], [15], [23], but they cannot be compared with the benchmark functions we have considered.

\section{BOOLEAN RELATIONS}

The concept of Boolean relations was introduced as a more general scheme for the non-deterministic specification of logic networks, which cannot always be represented using don't cares [1], [3].

Definition 1: A Boolean relation is a one-to-many multioutput Boolean mapping $\mathcal{R}:\{0,1\}^{n} \rightarrow\{0,1\}^{m}$, where $\{0,1\}^{n}$ and $\{0,1\}^{m}$ are called the input and output sets of $\mathcal{R}$.

A Boolean relation $\mathcal{R}$ can be considered a generalization of a Boolean function, where a point in the input set $\{0,1\}^{n}$ can be associated with several points in the output set $\{0,1\}^{m}$; indeed, because of the one-to-many nature of Boolean relations, there may be several equivalent outputs for a given input. For example, consider the mapping $\mathcal{R}:\{0,1\}^{2} \rightarrow\{0,1\}^{3}$ such that:

\begin{tabular}{c|c}
$x$ & $\mathcal{R}(x)$ \\
\hline 00 & $\{001,100\}$ \\
01 & $\{000\}$ \\
10 & $\{101,111\}$ \\
11 & $\{100,010,001\}$
\end{tabular}

Note that we cannot represent this mapping using a simple incompletely specified Boolean function, since, for example, the input 00 can have as output 001 or 100 that cannot be merged into a single cube. 

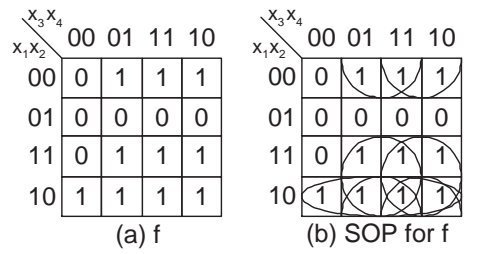

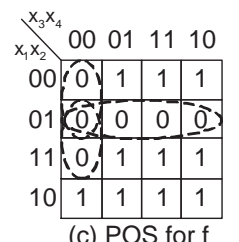

(c) POS for $f$

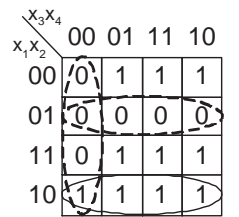

(d) Bi-cond. form for $\mathrm{f}$

Fig. 1. Example of Karnaugh maps of a SOP form (b) a POS form (c) and a circuit $f_{0} \Rightarrow f_{1} \equiv \bar{f}_{0}+f_{1}$ (d) for the Boolean function $f$ in (a).

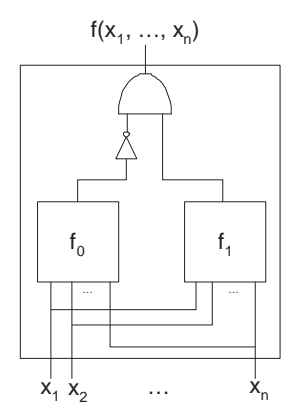

Fig. 2. Circuit decomposition with AND gate.

A relation $\mathcal{R}$ is well-defined if for all $x \in\{0,1\}^{n}$ there is $y \in\{0,1\}^{m}$ such that $(x, y) \in \mathcal{R}$. To any relation $\mathcal{R}$ we can associate a set $\mathcal{F}(\mathcal{R})$ of all compatible multi-output Boolean functions, i.e. the set of all functions $g$ such that, for all inputs $x \in\{0,1\}^{n}, g(x)$ is contained in the set $\mathcal{R}(x)$ of the outputs related to $x$. In this case, we write $g \subseteq \mathcal{R}$. For example, consider the mapping $\mathcal{R}$ described in the previous example. $\mathcal{F}(\mathcal{R})$ contains the following Boolean function $g:\{0,1\}^{n} \rightarrow\{0,1\}^{m}:$

\begin{tabular}{c|c}
$x$ & $g(x)$ \\
\hline 00 & 001 \\
01 & 000 \\
10 & 101 \\
11 & 100
\end{tabular}

The problem of the optimal implementation of a Boolean relation $\mathcal{R}$ is that of selecting, among the possible functions compatible with $\mathcal{R}$, one of minimum cost according to a given metric. More precisely, the solution of a Boolean relation $\mathcal{R}$ is a multi-output Boolean function $g \in \mathcal{F}(\mathcal{R})$. The function $g$ is an optimal solution of $\mathcal{R}$ according to a given cost function $\mu$, if for all $g^{\prime} \in \mathcal{F}(\mathcal{R}), \mu(g) \leq \mu\left(g^{\prime}\right)$. In this paper, we will consider as cost $\mu(g)$ the number of literals in a minimal SOP form for $f$.

\section{BI-DECOMPOSED CIRCUITS}

Given an incompletely specified function (ISF) $f$, defined as on-set $f_{o n}$, off-set $f_{o f f}$ and dc-set $f_{d c}$, we want to decompose $f$ in $u$ and $v$, such that $f$ is covered by $u$ op $v$, where $o p$ is a given binary operation, e.g. an AND, OR or XOR gate. The inputs of $u$ and $v$ are the same as the inputs of $f$. The output of $f$ is the output of the chosen gate $o p$ which takes in input $u\left(x_{1}, \ldots, x_{n}\right)$ and $v\left(x_{1}, \ldots, x_{n}\right)$. Figure 2 reports the circuit obtained when $o p$ is represented by the $\bar{u} v$ gate $(\notin)$.

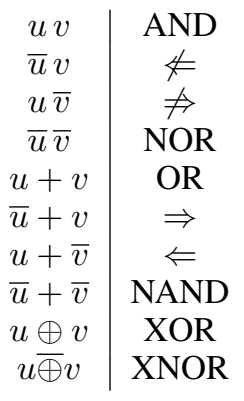

Fig. 3. Non-trivial binary operations

This problem can be formulated as that of solving Boolean relations. For each binary operation $o p$, we define a relation $\mathcal{R}_{o p}$ whose set of compatible functions $\mathcal{F}\left(\mathcal{R}_{o p}\right)$ corresponds exactly to the set of pairs $(u, v)$ occurring in all bi-decomposed circuit implementations of $f$ with respect to the chosen operation op. An optimal solution of $\mathcal{R}_{o p}$ is an optimal bidecomposed circuit for $f$.

Let $\mathcal{R}_{o p}:\{0,1\}^{n} \rightarrow\{0,1\}^{2}$ be a Boolean relation, describing all possible pairs of functions $u, v$ defining a bidecomposed circuit for $f$. We show how to construct the relation $\mathcal{R}_{o p}$ for any binary operation op for the ten (out of sixteen) binary operations that depend on both input variables and omit operations $1,0, u, \bar{u}, v, \bar{v}$. The 10 binary ops are shown in Figure 3. To construct $\mathcal{R}_{o p}$, three cases are distinguished, depending on whether the input vector $x \in\{0,1\}^{n}$ belongs to the on-set, the off-set, or the dc-set of the function $f$. We partition these ops into the first four, the second four and the last two. For the first four, $u v, \bar{u} v, u \bar{v}, \overline{u v}$, we note that each can be obtained from another by complementing one or more inputs. As an example, we construct $\mathcal{R}_{\sharp}$ as follows:

- all points $x \in f_{\text {on }}$ must be associated to the output 01 , so that the output of the circuit $\bar{u} v$ evaluates to 1 , thus we define $\mathcal{R}_{\nLeftarrow}(x)=\{01\}$;

- $\quad$ all points $x \in f_{\text {off }}$ must be associated to one of the three output values on which $\bar{u} v$ evaluates to 0 , thus we define $\mathcal{R}_{\sharp}(x)=\{00,10,11\}=\{1-,-0\}$;

- all points $x \in f_{d c}$ can be associated to any output, thus we have $\mathcal{R}_{\sharp}(x)=\{--\}$.

The relations $\mathcal{R}_{A N D}, \mathcal{R}_{\nexists}$, and $\mathcal{R}_{N O R}$, corresponding to the other three operations in the first group (the AND group), can be defined in an analogous way. These are summarized in Table I. Similarly, the four Boolean relations $\mathcal{R}_{O R}, \mathcal{R}_{\Rightarrow}, \mathcal{R}_{\Leftarrow}$, and $\mathcal{R}_{N A N D}$ (the OR group) are summarized in Table II and the last two (the XOR group) are summarized in Table III. 
TABLE I. AND TABLE

\begin{tabular}{|l|c|c|c|c|}
\hline & $\mathcal{R}_{\sharp}$ & $\mathcal{R}_{A N D}$ & NOR & $\mathcal{R}_{\nRightarrow}$ \\
\hline$x \in f_{\text {on }}$ & $\{01\}$ & $\{11\}$ & $\{00\}$ & $\{10\}$ \\
$x \in f_{\text {off }}$ & $\{1-,-0\}$ & $\{0-,-0\}$ & $\{1-,-1\}$ & $\{0-,-1\}$ \\
$x \in f_{d c}$ & $\{--\}$ & $\{--\}$ & $\{--\}$ & $\{--\}$ \\
\hline
\end{tabular}

TABLE II. OR TABLE

\begin{tabular}{|l|c|c|c|c|}
\hline & $\mathcal{R}_{\Rightarrow}$ & $\mathcal{R}_{O R}$ & $\mathcal{R}_{N A N D}$ & $\mathcal{R} \Leftarrow$ \\
\hline$x \in f_{\text {on }}$ & $\{0-,-1\}$ & $\{1-,-1\}$ & $\{0-,-0\}$ & $\{1-,-0\}$ \\
$x \in f_{\text {off }}$ & $\{10\}$ & $\{00\}$ & $\{11\}$ & $\{01\}$ \\
$x \in f_{\text {dc }}$ & $\{--\}$ & $\{--\}$ & $\{--\}$ & $\{--\}$ \\
\hline
\end{tabular}

Given an ISF, $f=\left(f_{o n}, f_{o f f}\right)$, the output $z$ of the LUT must satisfy $f_{o n} \subseteq z \subseteq \overline{f_{o f f}}$, i.e. $z$ is a cover of $f$. This constraint is guaranteed by the Boolean relation minimizer. Let $\left(f_{o n}^{1}, f_{o f f}^{1}\right)$ be an ISF associated with the first output $u$ of the PLA, and similarly $\left(f_{o n}^{2}, f_{o f f}^{2}\right)$ be that for the second output. Then either $u=u^{1}$ where $f_{o n}^{1} \subseteq u^{1} \subseteq \overline{f_{o f f}^{1}}$ or $u=u^{0}$ where $f_{o f f}^{1} \subseteq u^{0} \subseteq \overline{f_{o n}^{1}}$ and similarly for the second output, $v=v^{1}$ or $v=v^{0}$. Thus the LUT at the output affords us the flexibility of implementing an onset cover or offset cover for the two outputs leading to a two-output phase assignment problem.

Since our three-level form consists of a PLA with each pair of outputs feeding into a two-input LUT, only what is implemented in the PLA is important; any cost function should be independent of the $o p$ implemented in the LUT.

Note that the ISFs associated with $u$ and $v$ depend on the $o p$ in the LUT as dictated by the Boolean relations given by the three Tables I, II, III. Any entry (column) in a table can be obtained from any other entry in the same table by simply inverting one or more of the inputs. Thus for a given table, phase assignment maps one column into another. The three tables are distinguished by whether the offset is partitioned (AND table), the onset is partitioned (OR table) or both are partitioned (XOR table). For example, in the first table for $\mathcal{R}_{\sharp}$, the care minterms of $f$ are distributed as follows: $f_{\text {on }}$ is put in $f_{o f f}^{1}$ as well as in $f_{o n}^{2}$, and $f_{o f f}$ is partitioned into three parts, those in $f_{o n}^{1}$ only, those in $f_{o f f}^{2}$ only, and those in both. How this partitioning is done is the task of the Boolean relation minimizer. Thus, given a distribution of care minterms (i.e. partitionings), the four choices for the two outputs implemented in the PLA are $(u, v) \in\left\{\left(u^{1}, v^{1}\right),\left(u^{0}, v^{1}\right),\left(u^{1}, v^{0}\right),\left(u^{0}, v^{0}\right)\right\}$ and these choices correspond to the choices of Boolean relations in each of the three tables. We note for future reference, that for the Boolean relation minimizer, BREL [1], complementing an input in the Boolean relation will simply switch the implementation of that output of the PLA from a cover of the onset to a cover of the offset.

In the literature, the following methods for three level minimization have been investigated: 1) AND-OR-AND [8,11], 2) OR-AND-OR [7,17], and 3) AND-OR-XOR [5,6,9]. To

TABLE III. XOR TABLE

\begin{tabular}{|c|c|c|}
\hline & $\mathcal{R}_{X N O R}$ & $\mathcal{R}_{X O R}$ \\
\hline$x \in f_{\text {on }}$ & $\{00,11\}$ & $\{01,10\}$ \\
$x \in f_{\text {off }}$ & $\{01,10\}$ & $\{00,11\}$ \\
$x \in f_{d c}$ & $\{--\}$ & $\{--\}$ \\
\hline
\end{tabular}

TABLE V. GAINS OF BEST op CIRCUITS

\begin{tabular}{|r|rrr|rrr|}
\hline & \multicolumn{3}{|c|}{ EXACT } & \multicolumn{3}{c|}{ HEURISTIC } \\
\hline & $\notin$ & $\Rightarrow$ & XNOR & $\notin$ & $\Rightarrow$ & XNOR \\
\hline time & $2 \%$ & $4 \%$ & $2 \%$ & $11 \%$ & $11 \%$ & $8 \%$ \\
area & $89 \%$ & $87 \%$ & $78 \%$ & $65 \%$ & $63 \%$ & $47 \%$ \\
delay & $63 \%$ & $69 \%$ & $50 \%$ & $63 \%$ & $69 \%$ & $43 \%$ \\
\hline
\end{tabular}

TABLE VI. AVERAGE GAIN OF COMPLEMENTED CIRCUITS

\begin{tabular}{|c|rrr|rrr|}
\hline & \multicolumn{3}{|c|}{ EXACT } & \multicolumn{3}{c|}{ HEURISTIC } \\
\hline & $\notin$ & $\Rightarrow$ & XNOR & $\notin$ & $\Rightarrow$ & XNOR \\
\hline time & $-86 \%$ & $-83 \%$ & $-63 \%$ & $-1077 \%$ & $-853 \%$ & $-1030 \%$ \\
area & $29 \%$ & $29 \%$ & $26 \%$ & $16 \%$ & $16 \%$ & $13 \%$ \\
delay & $19 \%$ & $20 \%$ & $15 \%$ & $12 \%$ & $15 \%$ & $6 \%$ \\
\hline
\end{tabular}

compare their results against ours is difficult because each method uses a different minimizer which may induce different partitionings. Although a partitioning induced by any method can be inferred from $u$ and $v$ by determining which onset/offset minterms are in $u, \bar{u}, v, \bar{v}$ for any of the methods, it is not easy to force the algorithms to use the same partitionings. Thus a controlled experiment can't be done easily. However, we note that the AND-OR-AND method might be similar to using BREL on $\mathcal{R}_{A N D}$, OR-AND-OR similar to $\mathcal{R}_{O R}$, and AND-OR-XOR similar to $\mathcal{R}_{X O R}$. With the formalism of the Boolean relations, we can rephrase our complemented circuit minimization problem as the problem of finding an optimal implementation of $\mathcal{R}_{o p}$ (for $o p$ corresponding to one of the selected binary operations), that is, of selecting among all possible two-output functions compatible with $\mathcal{R}_{o p}$, the one defining the couple $(u, v)$ leading to a circuit of minimal cost, according to a given cost metric:

Theorem 1: The set $\mathcal{F}\left(\mathcal{R}_{o p}\right)$ of all two-output functions compatible with the relation $\mathcal{R}_{o p}$ specifies exactly the set of all pairs $(u, v)$, occurring in all possible circuit implementations where $z=u$ op $v$ is a cover of the ISF $f=\left(f_{o n}, f_{\text {off }}\right)$.

Proof: Let $C(f)$ be a circuit with two outputs $u$ and $v$, such that $z=u$ op $v$ is a cover of $f$. Then $C(f)$ is compatible with $R_{o p}$ because it is easy to verify that for all $x \in\{0,1\}^{n},(u(x), v(x)) \in \mathcal{R}_{o p}(x)$. Conversely, let $C(f)$ be any two-output function compatible with $\mathcal{R}_{o p}$. Observe that the definition of $R_{o p}$ guarantees that the two outputs of each function $g \in \mathcal{F}\left(\mathcal{R}_{o p}\right)$ combined with the chosen operation op, evaluates to 1 on all points in $f_{o n}$ and to 0 on all points in $f_{o f f}$. Thus, with $u$ as the first output of $g$ and $v$ as the second, we get that $u$ op $v$ is a cover of $f$.

Corollary 1: An optimum solution of the Boolean relation $\mathcal{R}_{o p}$, according to a given cost function $\mu$, defines an optimum bi-decomposed circuit, $z=u_{o p t}$ op $v_{\text {opt }}$ for $f$ with the minimum cost $\mu$.

Proof: An optimum bi-decomposed circuit for $f$ is one where $z=u_{o p t}$ op $v_{o p t}$ is a cover of $f$ and $\mu(z)$ is minimum. By Theorem 1, the two-output function $\left(u_{o p t}, v_{\text {opt }}\right)$ is compatible with $\mathcal{R}_{o p}$ and hence a solution of $\mathcal{R}_{o p}$. Since $\mu\left(u_{\text {opt }}, v_{\text {opt }}\right)$ is minimum then also $z=u_{o p t}$ op $v_{\text {opt }}$ is a minimum cover with respect to $o p$ and $\mu$

\section{EXPERIMENTAL RESULTS}

In this section we report the experimental results for the minimization of the Boolean relations of three op circuitss, one from each of the three Tables I, II, III. The representative 
TABLE IV. COMPARISON OF SOP VS op CIRCUITS

\begin{tabular}{|c|c|c|c|c|c|c|c|c|c|c|c|c|c|c|c|c|c|c|c|c|c|c|c|c|}
\hline \multirow[b]{2}{*}{ benchmark } & \multicolumn{3}{|c|}{ SOP exact } & \multicolumn{3}{|c|}{ SOP heuristic } & \multicolumn{3}{|c|}{$\notin$ exact } & \multicolumn{3}{|c|}{$\notin$ heuristic } & \multicolumn{3}{|c|}{$\Rightarrow$ exact } & \multicolumn{3}{|c|}{$\Rightarrow$ heuristic } & \multicolumn{3}{|c|}{ XNOR exact } & \multicolumn{3}{|c|}{ XNOR heuristic } \\
\hline & time & area & delay & time & area & delay & time & area & delay & time & area & delay & time & area & delay & time & area & delay & time & area & delay & time & area & delay \\
\hline add6 & 0.77 & 292 & 25.20 & 0.03 & 292 & 25.20 & 7.60 & 297 & 25.30 & 0.08 & 290 & 25.40 & 6.97 & 294 & 22.70 & 0.09 & 287 & 24.10 & 5.32 & 292 & 26.60 & 0.07 & 327 & 28.10 \\
\hline addm 4 & 0.04 & 934 & 42.70 & 0.02 & 959 & 41.90 & 7.31 & 781 & 30.10 & 0.02 & 918 & 38.50 & 7.51 & 807 & 36.90 & 0.02 & 899 & 39.60 & 8.10 & 745 & 31.00 & 0.02 & 1016 & 44.80 \\
\hline $\mathrm{al} 2$ & 0.86 & 340 & 15.10 & 0.00 & 340 & 15.10 & 3.76 & 332 & 14.00 & 0.01 & 335 & 14.20 & 0.58 & 331 & 13.10 & 0.00 & 443 & 13.80 & 4.87 & 505 & 16.10 & 0.01 & 425 & 15.10 \\
\hline alu2 & 0.01 & 176 & 16.40 & 0.00 & 176 & 14.50 & 1.71 & 160 & 12.30 & 0.03 & 203 & 20.50 & 1.68 & 158 & 14.00 & 0.01 & 195 & 16.50 & 1.81 & 155 & 17.80 & 0.02 & 187 & 19.90 \\
\hline alu3 & 0.01 & 187 & 16.80 & 0.00 & 157 & 13.30 & 1.66 & 158 & 12.60 & 0.03 & 199 & 20.80 & 1.69 & 157 & 13.30 & 0.02 & 199 & 17.00 & 1.75 & 159 & 17.80 & 0.01 & 179 & 19.10 \\
\hline and & 0.02 & 982 & 38.50 & 0.01 & 986 & 37.30 & 7.38 & 736 & 37.00 & 0.09 & 815 & 34.20 & 7.00 & 752 & 38.60 & 0.06 & 852 & 34.50 & 7.50 & 818 & 35.10 & 0.06 & 892 & 35.80 \\
\hline b2 & 0.08 & 3984 & 76.90 & 0.01 & 3957 & 73.00 & 168.31 & 3219 & 69.10 & 1.32 & 3597 & 69.70 & 170.34 & 3207 & 66.30 & 1.10 & 3492 & 68.70 & 151.47 & 3156 & 65.20 & 1.60 & 3567 & 76.30 \\
\hline b3 & 0.81 & 1126 & 45.00 & 0.03 & 1095 & 44.70 & 281.90 & 877 & 34.60 & 0.51 & 1250 & 36.20 & 279.14 & 853 & 35.80 & 0.47 & 1337 & 39.20 & 192.95 & 903 & 36.80 & 0.45 & 1048 & 34.70 \\
\hline b4 & 1.17 & 649 & 31.20 & 0.00 & 649 & 31.20 & 7.39 & 596 & 26.60 & 0.75 & 657 & 28.00 & 7.19 & 594 & 26.60 & 0.69 & 731 & 30.40 & 8.18 & 616 & 28.80 & 0.62 & 697 & 29.70 \\
\hline $\mathrm{bcc}$ & 0.03 & 5167 & 92.10 & 0.03 & 5150 & 93.30 & 66.16 & 3850 & 66.80 & 1.13 & 5334 & 94.60 & 84.34 & 3799 & 64.30 & 0.72 & 5195 & 93.80 & 66.51 & 3985 & 67.40 & 0.84 & 5224 & 92.90 \\
\hline bench & 0.01 & 172 & 15.00 & 0.00 & 144 & 12.10 & 0.97 & 98 & 12.10 & 0.00 & 120 & 14.00 & 0.62 & 98 & 11.30 & 0.00 & 110 & 11.70 & 1.03 & 116 & 15.00 & 0.00 & 141 & 15.10 \\
\hline br2 & 0.00 & 280 & 28.30 & 0.00 & 280 & 28.30 & 1.81 & 237 & 18.40 & 0.00 & 265 & 19.00 & 1.28 & 234 & 18.10 & 0.00 & 261 & 25.10 & 2.04 & 256 & 21.70 & 0.00 & 268 & 24.90 \\
\hline $\mathrm{dc} 1$ & 0.00 & 85 & 11.80 & 0.00 & 85 & 12.10 & 0.69 & 72 & 10.30 & 0.00 & 77 & 13.40 & 0.41 & 74 & 10.80 & 0.00 & 79 & 13.80 & 0.74 & 80 & 11.60 & 0.00 & 109 & 15.40 \\
\hline dc2 & 0.00 & 243 & 24.70 & 0.00 & 233 & 20.40 & 1.46 & 184 & 20.30 & 0.00 & 210 & 18.30 & 1.59 & 185 & 19.40 & 0.00 & 199 & 18.20 & 1.42 & 197 & 20.50 & 0.00 & 220 & 21.10 \\
\hline ex7 & 0.14 & 225 & 21.20 & 0.00 & 225 & 21.20 & 3.06 & 194 & 24.90 & 0.04 & 231 & 20.10 & 2.78 & 193 & 19.70 & 0.04 & 238 & 20.60 & 3.37 & 219 & 23.00 & 0.04 & 251 & 24.00 \\
\hline exep & 0.04 & 1285 & 31.40 & 0.01 & 1275 & 36.10 & 9.18 & 1290 & 31.00 & 0.46 & 1295 & 33.50 & 1.86 & 1271 & 31.20 & 0.10 & 1300 & 31.40 & 11.08 & 1454 & 36.60 & 0.56 & 1311 & 36.90 \\
\hline exps & 0.06 & 3579 & 94.50 & 0.02 & 3549 & 101.10 & 17.69 & 2405 & 62.50 & 0.01 & 2697 & 73.50 & 17.98 & 2387 & 64.30 & 0.01 & 2574 & 71.90 & 16.87 & 2429 & 65.10 & 0.00 & 2713 & 68.20 \\
\hline fout & 0.05 & 535 & 30.40 & 0.00 & 472 & 27.10 & 2.82 & 339 & 23.10 & 0.00 & 398 & 25.00 & 2.80 & 336 & 21.90 & 0.00 & 418 & 26.20 & 2.40 & 325 & 23.10 & 0.00 & 402 & 28.00 \\
\hline in 1 & 0.07 & 3984 & 76.90 & 0.01 & 3957 & 73.00 & 167.55 & 3219 & 69.10 & 1.28 & 3597 & 69.70 & 170.23 & 3207 & 66.30 & 1.11 & 3492 & 68.70 & 151.62 & 3156 & 65.20 & 1.56 & 3567 & 76.30 \\
\hline in 3 & 0.12 & 1111 & 34.10 & 0.00 & 1111 & 34.10 & 8.71 & 928 & 32.80 & 0.16 & 985 & 32.80 & 7.79 & 913 & 32.90 & 0.22 & 970 & 33.60 & 8.95 & 971 & 36.20 & 0.08 & 974 & 37.80 \\
\hline in 4 & 0.65 & 1127 & 44.90 & 0.02 & 1077 & 44.80 & 286.03 & 924 & 35.60 & 0.59 & 1297 & 36.60 & 280.34 & 900 & 36.80 & 0.51 & 1384 & 39.30 & 194.52 & 950 & 37.80 & 0.46 & 1114 & 35.60 \\
\hline in6 & 0.77 & 662 & 31.30 & 0.00 & 662 & 31.30 & 7.06 & 609 & 26.70 & 0.23 & 669 & 28.30 & 6.64 & 607 & 26.70 & 0.14 & 744 & 30.50 & 7.67 & 629 & 28.80 & 0.08 & 710 & 29.80 \\
\hline in7 & 0.25 & 319 & 23.60 & 0.00 & 319 & 23.60 & 4.80 & 305 & 23.30 & 0.13 & 340 & 26.90 & 4.81 & 304 & 23.10 & 0.09 & 325 & 28.40 & 4.99 & 338 & 27.10 & 0.09 & 379 & 33.50 \\
\hline luc & 0.00 & 821 & 40.70 & 0.00 & 845 & 40.20 & 5.40 & 596 & 28.00 & 0.01 & 649 & 31.80 & 4.66 & 599 & 27.30 & 0.01 & 641 & 28.20 & 4.71 & 620 & & 0.00 & 727 & 34.00 \\
\hline $\mathrm{m} 181$ & 0.24 & 234 & 20.50 & 0.00 & 174 & 16.40 & 0.92 & 114 & 11.10 & 0.00 & 141 & 18.10 & 1.08 & 113 & 12.00 & 0.00 & 127 & 11.50 & 1.34 & 134 & 14.80 & 0.00 & 169 & 19.30 \\
\hline $\mathrm{m} 3$ & 0.00 & 1169 & 47.10 & 0.00 & 1269 & 51.5 & 3.96 & 502 & 29.90 & 0.00 & 547 & 28.00 & & 492 & 30.60 & 0.01 & 536 & 30. & 3.67 & 535 & 33.60 & 0.00 & 531 & 31.30 \\
\hline $\mathrm{m} 4$ & 0.04 & 1904 & 66.50 & 0.02 & 1778 & 54.20 & 5.91 & 799 & 35.20 & 0.00 & 863 & 36.50 & 5.80 & 807 & 34.80 & 0.02 & 867 & 36.90 & 5.64 & 812 & 38.50 & 0.01 & 901 & 38.70 \\
\hline mlp4 & 0.10 & 702 & 34.50 & 0.01 & 686 & 36.4 & 4.69 & 569 & 30.50 & 0.01 & 629 & 30.1 & 4.5 & 579 & 31.10 & 0.00 & 595 & 29.3 & 4.64 & 573 & 10 & 0.01 & 630 & 36.90 \\
\hline $\mathrm{mp} 2 \mathrm{~d}$ & 0.08 & 268 & 20.60 & 0.00 & 251 & 19.80 & 1.72 & 224 & 17.00 & 0.00 & 269 & 20.30 & 1.36 & 222 & 19.00 & 0.00 & 233 & 17.20 & 2.17 & 239 & 17.10 & 0.00 & 238 & 15.20 \\
\hline & 0.03 & 651 & 32.20 & 0.02 & 645 & 36.00 & 3.63 & 382 & 24.60 & 0.00 & 466 & 28.5 & & 375 & 21.20 & 0.00 & 441 & 27.80 & 3.74 & 396 & & 0.02 & 478 & 26.80 \\
\hline p3 & 0.02 & 447 & 26.70 & 0.00 & 448 & 26.60 & 2.35 & 245 & 21.50 & 0.00 & 293 & 23.90 & 2.32 & 248 & 18.70 & 0.01 & 285 & 19.10 & 2.40 & 258 & 21.50 & 0.00 & 308 & 23.50 \\
\hline shift & 801.94 & 372 & 27.00 & 0.00 & 372 & & 3.64 & 371 & 27.20 & 0.03 & & & & 372 & & & 372 & 27.00 & 4.17 & 377 & & 0.01 & 372 & \\
\hline spla & 0.80 & 2422 & 57.40 & 0.19 & 2470 & 68.60 & 14.56 & 1678 & 44.70 & 0.05 & 1859 & 48.40 & 14.38 & 1688 & 46.30 & 0.09 & 1862 & 47.40 & 16.68 & 1763 & 40.60 & 0.04 & 1932 & 49.00 \\
\hline sym10 & 1.23 & 519 & 29.90 & 0.02 & 592 & 31.70 & 59.06 & 344 & 35.70 & 0.00 & 379 & 31.50 & 54.97 & 301 & 32.40 & 0.01 & 350 & 27.50 & 32.66 & 301 & & 0.01 & 379 & 31.50 \\
\hline $\mathrm{t} 1$ & 6.83 & 546 & 22.10 & 0.01 & 458 & 21.90 & 3.66 & 347 & 18.00 & 0.00 & 378 & 19.30 & 3.46 & 353 & 17.90 & 0.03 & 388 & 20.50 & 4.49 & 374 & 19.30 & 0.00 & 412 & 22.90 \\
\hline $\mathrm{t} 4$ & 0.01 & 102 & 14.10 & 0.00 & 96 & & 0.91 & 96 & 14.90 & 0.00 & 97 & & & 93 & & & 100 & & 0.97 & 114 & & 0.00 & 116 & 19.30 \\
\hline test 1 & 39.11 & 1392 & 47.50 & 0.02 & 1318 & 46.60 & 7.95 & 935 & 39.40 & 0.10 & 1351 & 44.90 & 8.37 & 935 & 35.30 & 0.07 & 1369 & 47.00 & 8.00 & 944 & 39.00 & 0.10 & 1302 & 46.30 \\
\hline tial & 1.31 & 2376 & 68.70 & 0.20 & 2327 & 66.00 & 123.41 & 1516 & 51.60 & 0.51 & 1654 & 50.40 & 104.32 & 1489 & 50.80 & 0.31 & 1715 & 49.30 & 142.05 & 1537 & 53.10 & 0.64 & 2428 & 60.00 \\
\hline vg2 & 0.07 & 341 & 18.60 & 0.00 & 341 & 18.60 & 3.80 & 287 & 22.00 & 0.10 & 495 & 21.70 & 4.24 & 284 & 20.00 & 0.09 & 369 & 18.60 & 5.16 & 292 & 22.00 & 0.06 & 376 & 18.60 \\
\hline vtx1 & 0.05 & 324 & 21.30 & 0.00 & 324 & 21.30 & 3.19 & 238 & 17.90 & 0.07 & 424 & 28.30 & 3.46 & 259 & 20.50 & 0.10 & 383 & 25.30 & 4.13 & 257 & 19.70 & 0.05 & 429 & 27.20 \\
\hline x6dn & 0.04 & 789 & 34.40 & 0.00 & 762 & 31.20 & 6.91 & 725 & 36.40 & 0.13 & 772 & 32.20 & 6.91 & 738 & 32.50 & 0.13 & 770 & 33.50 & 6.75 & 734 & 32.90 & 0.08 & 777 & 34.80 \\
\hline $\mathrm{x} 9 \mathrm{dn}$ & 0.06 & 384 & 23.00 & 0.00 & 384 & 23.00 & 3.33 & 262 & 22.70 & 0.08 & 530 & 28.00 & 4.90 & 254 & 20.90 & 0.12 & 464 & 24.60 & 4.68 & 258 & 19.40 & 0.09 & 590 & 32.70 \\
\hline
\end{tabular}

TABLE VII. MinimizATION RESUlts

\begin{tabular}{|c|c|c|c|c|c|c|c|c|c|c|c|c|c|c|c|c|c|}
\hline \multirow[b]{2}{*}{ benchmark } & \multirow[b]{2}{*}{ in/out } & \multicolumn{2}{|c|}{$\notin$ exact } & \multicolumn{2}{|c|}{$\notin$ heuristic } & \multicolumn{2}{|c|}{$\Rightarrow$ exact } & \multicolumn{2}{|c|}{$\Rightarrow$ heuristic } & \multicolumn{2}{|c|}{ XNOR exact } & \multicolumn{2}{|c|}{ XNOR heuristic } & \multicolumn{2}{|c|}{ [9] } & \multicolumn{2}{|c|}{$[10]$} \\
\hline & & time & lit & time & lit & time & lit & time & lit & time & lit & time & lit & time & lit & time & lit \\
\hline $5 \times 1$ & $7 / 10$ & 0.00 & 17 & 0.00 & 17 & 0.00 & 20 & 0.00 & 20 & 0.00 & 20 & 0.00 & 20 & 14.00 & 55 & 14.80 & 42 \\
\hline 9sym & $9 / 1$ & 7.79 & 73 & 0.00 & 73 & 7.21 & 71 & 0.00 & 72 & 7.40 & 72 & 0.00 & 72 & & & 1.70 & 73 \\
\hline alu2 & $10 / 8$ & 1.71 & 67 & 0.03 & 79 & 1.68 & 68 & 0.01 & 83 & 1.81 & 48 & 0.02 & 64 & 32.00 & 52 & & \\
\hline alu3 & $10 / 8$ & 1.66 & 67 & 0.03 & 79 & 1.69 & 70 & 0.02 & 86 & 1.75 & 49 & 0.01 & 64 & 24.00 & 47 & & \\
\hline alu4 & $14 / 8$ & 107.70 & 518 & 0.41 & 631 & 101.58 & 520 & 0.27 & 632 & 87.42 & 472 & 0.53 & 658 & & & 131.90 & 447 \\
\hline b12 & $15 / 9$ & 0.82 & 42 & 0.01 & 51 & 0.96 & 40 & 0.00 & 47 & 1.28 & 42 & 0.00 & 58 & 30.00 & 28 & 9.10 & 31 \\
\hline bw & $5 / 28$ & 0.00 & 56 & 0.00 & 56 & 0.00 & 56 & 0.00 & 56 & 0.00 & 56 & 0.00 & 56 & & & 25.40 & 24 \\
\hline clip & $9 / 5$ & 3.60 & 149 & 0.01 & 158 & 3.55 & 150 & 0.00 & 159 & 3.71 & 116 & 0.03 & 162 & & & 10.50 & 95 \\
\hline con1 & $7 / 2$ & 0.18 & 10 & 0.00 & 10 & 0.25 & 10 & 0.00 & 11 & 0.29 & 10 & 0.00 & 13 & & & 1.00 & 9 \\
\hline cordic & $23 / 2$ & 1909.46 & 345 & 0.15 & 1180 & 1908.45 & 243 & 0.14 & 2058 & 1024.40 & 158 & 0.15 & 1187 & & & 231.80 & 156 \\
\hline dist & $8 / 5$ & 5.12 & 153 & 0.01 & 173 & 5.08 & 153 & 0.01 & 179 & 4.64 & 146 & 0.01 & 149 & 170.00 & 108 & & \\
\hline ex1010 & $10 / 10$ & 35.51 & 639 & 0.26 & 1118 & 35.47 & 630 & 0.27 & 1124 & 34.99 & 608 & 0.49 & 1051 & & & 109.70 & 725 \\
\hline & $7 / 9$ & 1.26 & 58 & 0.00 & 64 & 1.25 & 57 & 0.00 & 59 & 1.44 & 55 & 0.00 & 59 & & & 6.50 & 33 \\
\hline misex 1 & $8 / 7$ & 1.04 & 43 & 0.00 & 48 & 0.89 & 43 & 0.00 & 44 & 0.97 & 40 & 0.00 & 46 & & & 11.50 & 13 \\
\hline misex 2 & $25 / 18$ & 1.67 & 76 & 0.00 & 133 & 0.32 & 104 & 0.00 & 97 & 2.29 & 116 & 0.00 & 121 & & & 6.00 & 28 \\
\hline $\operatorname{misex} 3$ & $14 / 14$ & 106.90 & 585 & 0.44 & 1051 & 116.77 & 576 & 0.29 & 961 & 84.78 & 672 & 0.49 & 1270 & & & 112.00 & 191 \\
\hline newapla2 & $6 / 7$ & 0.52 & 30 & 0.00 & 41 & 0.00 & 42 & 0.00 & 42 & 0.63 & 42 & 0.00 & 34 & 0.39 & 5 & & \\
\hline newbyte & $5 / 8$ & 0.59 & 25 & 0.00 & 35 & 0.00 & 40 & 0.00 & 40 & 0.71 & 40 & 0.00 & 37 & 0.52 & 5 & & \\
\hline newcpla1 & $9 / 16$ & 1.85 & 81 & 0.00 & 94 & 1.41 & 81 & 0.01 & 95 & 2.30 & 99 & 0.00 & 92 & 26.00 & 27 & & \\
\hline newtpla & $15 / 5$ & 0.99 & 33 & 0.01 & 50 & 0.74 & 36 & 0.00 & 54 & 1.04 & 40 & 0.00 & 38 & 1.20 & 19 & & \\
\hline radd & $8 / 5$ & 1.54 & 76 & 0.01 & 86 & 1.43 & 77 & 0.00 & 75 & 1.18 & 49 & 0.00 & 52 & 14.00 & 39 & & \\
\hline rd53 & $5 / 3$ & 0.00 & 11 & 0.00 & 11 & 0.09 & 6 & 0.00 & 8 & 0.17 & 8 & 0.00 & 7 & 5.90 & 26 & 15.70 & 19 \\
\hline rd73 & $7 / 3$ & 0.00 & 17 & 0.00 & 17 & 0.16 & 6 & 0.00 & 9 & 0.24 & 8 & 0.00 & 9 & 312.00 & 79 & 25.50 & 83 \\
\hline rd84 & $8 / 4$ & 14.74 & 309 & 0.00 & 299 & 14.16 & 310 & 0.00 & 303 & 10.53 & 194 & 0.00 & 247 & & & 61.10 & 192 \\
\hline ryy6 & $16 / 1$ & 0.52 & 8 & 0.01 & 8 & 0.74 & 7 & 0.01 & 7 & 0.68 & 7 & 0.00 & 113 & 379.00 & 7 & & \\
\hline sao 2 & $10 / 4$ & 0.04 & 30 & 0.00 & 12 & 0.46 & 13 & 0.00 & 14 & 0.55 & 8 & 0.00 & 8 & & & 3.70 & 38 \\
\hline sqn & $7 / 3$ & 1.18 & 47 & 0.00 & 51 & 1.23 & 41 & 0.00 & 48 & 1.25 & 43 & 0.00 & 53 & 6.10 & 34 & & \\
\hline squar5 & $5 / 8$ & 0.69 & 36 & 0.00 & 37 & 0.61 & 39 & 0.00 & 39 & 0.85 & 38 & 0.00 & 43 & & & 4.40 & 22 \\
\hline t2 & $17 / 16$ & 2.69 & 94 & 0.02 & 126 & 2.42 & 124 & 0.01 & 123 & 3.06 & 110 & 0.00 & 116 & 44.00 & 46 & & \\
\hline $\mathrm{t} 481$ & $16 / 1$ & 14.69 & 361 & 0.01 & 361 & 12.24 & 360 & 0.00 & 360 & 43.92 & 441 & 0.01 & 482 & & & 557.20 & 113 \\
\hline table 3 & $14 / 14$ & 62.89 & 751 & 0.58 & 1385 & 59.19 & 861 & 0.31 & 1094 & 57.80 & 838 & 0.46 & 1140 & & & 79.30 & 176 \\
\hline table5 & $17 / 15$ & 68.56 & 862 & 0.48 & 1427 & 72.28 & 977 & 0.35 & 797 & 67.98 & 800 & 0.41 & 1178 & & & 100.70 & 158 \\
\hline x $1 \mathrm{dn}$ & $27 / 6$ & 2.27 & 78 & 0.11 & 83 & 2.58 & 76 & 0.10 & 81 & 5.83 & 76 & 0.19 & 81 & 237.00 & 81 & & \\
\hline $\mathrm{x} 9 \mathrm{dn}$ & $27 / 7$ & 3.33 & 111 & 0.08 & 244 & 4.90 & 103 & 0.12 & 158 & 4.68 & 110 & 0.09 & 297 & 307.00 & 81 & & \\
\hline xor5 & $5 / 1$ & 0.29 & 16 & 0.00 & 16 & 0.30 & 16 & 0.00 & 16 & 0.18 & 9 & 0.00 & 9 & & & 10.30 & 10 \\
\hline vg2 & $25 / 8$ & 0.61 & 22 & 0.13 & 42 & 0.63 & 18 & 0.15 & 22 & 0.71 & 18 & 0.21 & 21 & 366.00 & 88 & 43.40 & 102 \\
\hline $\mathrm{z} 4$ & $7 / 4$ & 1.09 & 55 & 0.00 & 61 & 1.12 & 62 & 0.00 & 60 & 0.95 & 41 & 0.00 & 41 & 2.10 & 33 & & \\
\hline Z5xp1 & $7 / 10$ & 1.69 & 86 & 0.00 & 82 & 1.76 & 79 & 0.00 & 83 & 1.53 & 74 & 0.00 & 80 & 112.00 & 56 & & \\
\hline
\end{tabular}


TABLE VIII. TIME, AREA AND DELAY: op CIRCUITS VS ESPRESSO WITH OUTPUT PHASE ASSIGNMENT.

\begin{tabular}{|c|c|c|c|c|c|c|c|c|c|c|c|c|c|c|c|c|c|c|c|c|c|c|c|c|}
\hline & \multicolumn{3}{|c|}{ ESPRESSO - exact } & \multicolumn{3}{|c|}{ ESPRESSO - heuristic } & \multicolumn{3}{|c|}{$\notin$ exact } & \multicolumn{3}{|c|}{$\notin$ heuristic } & \multicolumn{3}{|c|}{$\Rightarrow$ exact } & \multicolumn{3}{|c|}{$\Rightarrow$ heuristic } & \multicolumn{3}{|c|}{ XNOR exact } & \multicolumn{3}{|c|}{ XNOR heuristic } \\
\hline benchmark & time & area & delay & time & area & delay & time & area & delay & time & area & delay & time & area & delay & time & area & delay & time & area & delay & time & area & delay \\
\hline & 0.96 & 292 & 25.20 & 0.33 & 288 & 23.50 & 7.60 & 297 & 25.30 & 0.08 & 290 & 25.40 & 6.97 & 294 & 22.70 & 0.09 & 287 & 24.10 & 5.32 & 292 & 26.60 & 0.07 & 327 & 28.10 \\
\hline al2 & 1.09 & 340 & 15.10 & 0.05 & 370 & 15.20 & 3.76 & 332 & 14.00 & 0.01 & 335 & 14.20 & 0.58 & 331 & 13.10 & 0.00 & 443 & 13.80 & 4.87 & 505 & 16.10 & 0.01 & 425 & 15.10 \\
\hline alcom & 0.38 & 222 & 12.30 & 0.00 & 231 & 12.30 & 2.08 & 210 & 12.20 & 0.02 & 210 & 11.20 & 0.21 & 210 & 11.20 & 0.01 & 216 & 11.10 & 3.25 & 345 & 16.50 & 0.01 & 304 & 15.40 \\
\hline alu1 & 0.03 & 53 & 6.80 & 0.00 & 55 & 6.80 & 0.21 & 53 & 6.80 & 0.00 & 53 & 6.80 & 0.06 & 53 & 6.80 & 0.00 & 53 & 6.80 & 0.62 & 59 & 7.00 & 0.00 & 85 & 12.00 \\
\hline b10 & 0.10 & 876 & 42.80 & 0.09 & 881 & 45.20 & 10.40 & 721 & 31.90 & 0.13 & 827 & 34.80 & 11.47 & 723 & 32.00 & 0.11 & 857 & 37.00 & 10.00 & 745 & 37.00 & 0.15 & 909 & 37.30 \\
\hline b3 & 1.12 & 1126 & 45.00 & 1.03 & 1095 & 44.70 & 281.90 & 877 & 34.60 & 0.51 & 1250 & 36.20 & 279.14 & 853 & 35.80 & 0.47 & 1337 & 39.20 & 192.95 & 903 & 36.80 & 0.45 & 1048 & 34.70 \\
\hline b4 & 1.62 & 649 & 31.20 & 1.64 & 649 & 31.20 & 7.39 & 596 & 26.60 & 0.75 & 657 & 28.00 & 7.19 & 594 & 26.60 & 0.69 & 731 & 30.40 & 8.18 & 616 & 28.80 & 0.62 & 697 & 29.70 \\
\hline b7 & 0.00 & 234 & 16.30 & 0.01 & 219 & 15.90 & 1.93 & 195 & 15.00 & 0.01 & 212 & 16.60 & 0.22 & 196 & 15.30 & 0.00 & 203 & 16.10 & 2.72 & 294 & 18.10 & 0.00 & 236 & 17.10 \\
\hline b9 & 0.20 & 225 & 21.20 & 0.04 & 225 & 21.20 & 3.14 & 194 & 24.90 & 0.04 & 231 & 20.10 & 2.80 & 193 & 19.70 & 0.03 & 238 & 20.60 & 3.36 & 219 & 23.00 & 0.05 & 251 & 24.00 \\
\hline bench & 0.01 & 172 & 15.00 & 0.00 & 218 & 20.50 & 0.97 & 98 & 12.10 & 0.00 & 120 & 14.00 & 0.62 & 98 & 11.30 & 0.00 & 110 & 11.70 & 1.03 & 116 & 15.00 & 0.00 & 141 & 15.10 \\
\hline dist & 0.01 & 735 & 36.70 & 0.05 & 790 & 40.40 & 5.12 & 596 & 33.40 & 0.01 & 646 & 35.20 & 5.08 & 611 & 32.50 & 0.01 & 625 & 29.90 & 4.64 & 543 & & & 600 & 37.90 \\
\hline fout & 0.06 & 535 & 30.40 & 0.02 & 519 & 29.40 & 2.82 & 339 & 23.10 & 0.00 & 398 & 25.00 & 2.80 & 336 & 21.90 & 0.00 & 418 & 26.20 & 2.40 & 325 & 23.10 & 0.00 & 402 & 28.00 \\
\hline in 0 & 0.03 & 1005 & 47.80 & 0.07 & 1032 & 54.20 & 12.44 & 835 & 36.60 & 0.17 & 979 & 41.40 & 13.36 & 841 & 38.30 & 0.13 & 988 & 39.40 & 12.03 & 864 & 38.40 & 0.14 & 1161 & 46.50 \\
\hline in 1 & 0.09 & 3984 & 76.90 & 0.16 & 3957 & 73.00 & 167.55 & 3219 & 69.10 & 1.28 & 3597 & 69.70 & 170.23 & 3207 & 66.30 & 1.11 & 3492 & 68.70 & 151.62 & 3156 & 65.20 & 1.56 & 3567 & 76.30 \\
\hline in 2 & 0.02 & 939 & 36.70 & 0.12 & 997 & 40.80 & 88.78 & 831 & 34.30 & 0.45 & 953 & 37.60 & 104.00 & 820 & 34.90 & 0.38 & 1019 & 37.50 & 86.56 & 847 & 40.50 & 0.38 & 982 & 37.10 \\
\hline in 3 & 0.16 & 1111 & 34.10 & 0.28 & 1095 & 34.60 & 8.71 & 928 & 32.80 & 0.16 & 985 & 32.80 & 7.79 & 913 & 32.90 & 0.22 & 970 & 33.60 & 8.95 & 971 & 36.20 & 0.08 & 974 & 37.80 \\
\hline in 4 & 0.84 & 1127 & 44.90 & 1.04 & 1128 & 44.00 & 286.03 & 924 & 35.60 & 0.59 & 1297 & 36.60 & 280.34 & 900 & 36.80 & 0.51 & 1384 & 39.30 & 194.52 & 950 & & 0.46 & 1114 & 35.60 \\
\hline in5 & 0.06 & 865 & 38.50 & 0.28 & 865 & 38.50 & 8.47 & 686 & 31.90 & 0.15 & 937 & 34.90 & 8.47 & 696 & 31.40 & 0.18 & 831 & 31.70 & 11.35 & 720 & 31.60 & 0.21 & 954 & 40.70 \\
\hline in6 & 0.99 & 662 & 31.30 & 0.48 & 662 & 31.30 & 7.06 & 609 & 26.70 & 0.23 & 669 & 28.30 & 6.64 & 607 & 26.70 & 0.14 & 744 & 30.50 & 7.67 & 629 & 28.80 & 0.08 & 710 & 29.80 \\
\hline in7 & 0.32 & 319 & 23.60 & 0.03 & 321 & 23.90 & 4.80 & 305 & 23.30 & 0.13 & 340 & 26.90 & 4.81 & 304 & 23.10 & 0.09 & 325 & 28.40 & 4.99 & 338 & 27.10 & 0.09 & 379 & 33.50 \\
\hline $\operatorname{lin}$ & 3.06 & 2849 & 89.40 & 0.24 & 3289 & 98.50 & 15.26 & 1755 & 53. & 0.02 & 1909 & 58.50 & 14.11 & 1757 & 52.30 & 0.01 & 1899 & 54.80 & 13.47 & 1645 & 54.30 & 0.01 & 1921 & 54.40 \\
\hline $\mathrm{m} 181$ & 0.30 & 234 & 20.50 & 0.02 & 132 & 12.90 & 0.92 & 114 & 11.10 & 0.00 & 141 & 18.10 & 1.08 & 113 & 12.6 & 0.00 & 127 & & 1.34 & 134 & 14.80 & 0.00 & 169 & 19.30 \\
\hline & 0.04 & 1904 & 66.5 & 0.09 & 1166 & 44.80 & 5.91 & 799 & 35.20 & 0.00 & 863 & 36.50 & 5.80 & 807 & 34.8 & 0.02 & 867 & 36.90 & 5.64 & 812 & 38.50 & 0.01 & 901 & 38.70 \\
\hline $\max 128$ & 0.03 & 2149 & 67.7 & 0.07 & 1588 & 55.80 & 5.08 & 632 & 30.90 & 0.01 & 701 & 34. & 4.89 & 624 & 31.4 & 0.00 & 698 & 33.00 & 4.71 & 625 & 30.50 & 0.00 & 683 & 38.00 \\
\hline $\max 512$ & 0.04 & 919 & 41.0 & 0.11 & 793 & 38.80 & 6.74 & 664 & 36. & 0.02 & 780 & 44 & 6.59 & 669 & 35. & 0.02 & 772 & 43. & 6.00 & 659 & 33 & 0.01 & 753 & 43.50 \\
\hline & 0.12 & 702 & 34.50 & 0.08 & 709 & 35.70 & 4.69 & 569 & 30. & 0.01 & 629 & 30.1 & 4.59 & 579 & 31.1 & 0.00 & 595 & 29. & 4.64 & 573 & 35. & 0.01 & 630 & 36.90 \\
\hline $\mathrm{mp} 2 \mathrm{~d}$ & 0.10 & 268 & 20.60 & 0.04 & 227 & 17.40 & 1.72 & 224 & 17.00 & 0.00 & 269 & 20.30 & 1.36 & 222 & 19.00 & 0.00 & 233 & 17.20 & 2.17 & 239 & 17.10 & 0.00 & 238 & 15.20 \\
\hline spla & 0.96 & 2422 & 57.40 & 0.96 & 2 & 0.00 & 14.56 & 1678 & 44.70 & 0.05 & 1859 & 48.40 & 14.38 & 1688 & 46.30 & 0.09 & 1862 & 47.40 & 16.68 & 1763 & 40. & 0.04 & 1932 & 49.00 \\
\hline sym10 & 1.66 & 519 & 29. & 0.0 & 592 & 31.7 & 59.06 & 34 & 35 . & 0.00 & 379 & 31. & 54.97 & 30 & 32. & & 35 & 27. & 32.6 & 301 & 30 & & 37 & 31.50 \\
\hline t1 & 8.86 & 546 & 22. & 0.2 & 487 & 21. & 3.66 & 347 & 18. & 0.00 & 378 & 19 & 3. & 353 & 17. & & 38 & 20. & 4.49 & 374 & 19 & 0.00 & 412 & 22.90 \\
\hline t2 & 0.00 & 352 & 24.10 & 0.08 & 351 & 26. & 2.69 & 281 & & 0.02 & 300 & 20.4 & 2.42 & 283 & 20. & 0.01 & 30 & 20.70 & 3.06 & 314 & 19. & 0.00 & 322 & 20.40 \\
\hline $\mathrm{t} 4$ & 0.01 & 102 & 14.10 & 0.03 & 92 & 12.20 & 0.91 & 96 & 14.90 & 0.00 & 97 & 14.80 & 0.63 & 93 & 13.00 & 0.00 & 100 & 9.40 & 0.97 & 114 & 11.90 & 0.00 & 116 & 19.30 \\
\hline test1 & 50.63 & 1392 & 47.50 & 0.18 & 1474 & 49.70 & 7.95 & 935 & 39.40 & 0.10 & 1351 & 44.90 & 8.37 & 935 & 35.30 & 0.07 & $\begin{array}{l}1369 \\
\end{array}$ & 47.00 & 8.00 & 944 & 39.00 & 0.10 & 1302 & 46.30 \\
\hline tial & 1.68 & 2376 & 68.7 & 1.01 & 1928 & 49 & 123.41 & 1516 & & & 1654 & 50. & 104.32 & 1489 & & & 171 & 49 & 142.05 & 1537 & & 0.64 & 2428 & 60.00 \\
\hline vg2 & 0.14 & 46 & 10 & 0.01 & 46 & 10. & 3.8 & 28 & & 0. & 4 & & 4. & 28 & 20 & & 36 & 18 & 5. & 29 & & 0.0 & & 18.60 \\
\hline vtx 1 & 0.08 & 324 & 21.30 & 0.43 & 324 & 21. & 3.19 & 238 & 17 & 0.07 & 42 & 28.3 & 3.46 & 25 & 20. & 0.10 & 383 & 25. & 4.13 & 257 & 19.70 & 0.05 & 429 & 27.20 \\
\hline x6dn & 0.06 & 789 & 34.40 & 0.12 & 762 & 31.20 & 6.91 & 725 & 36.40 & 0.13 & 772 & 32.20 & 6.91 & 738 & 32.50 & 0.13 & 770 & 33.50 & 6.75 & 734 & 32.90 & 0.08 & 777 & 34.80 \\
\hline $\mathrm{x} 9 \mathrm{ddn}$ & 0.08 & 384 & 23.00 & 0.64 & 545 & 37.70 & 3.33 & 262 & 22.70 & 0.08 & 530 & 28.00 & 4.90 & 254 & 20.90 & 0.12 & 464 & 24.60 & 4.68 & 258 & 19.40 & 0.09 & 590 & 32.70 \\
\hline
\end{tabular}

circuits have at least one complemented input to distinguish them from existing published methods. We report the result for the $o p$ gates $\nLeftarrow, \Rightarrow$, and XNOR.

The algorithms have been implemented in $\mathrm{C}$, using the CUDD library for OBDDs to represent Boolean functions. We used BREL [1] for the synthesis of Boolean relations, because it finds better solutions in shorter runtimes than previous methods. The exact minimization in BREL is obtained using SIS; the heuristic minimization is based on the BDD size; even if the resulting number of literals is higher, the second version is useful since it is much more efficient w.r.t. synthesis time. The experiments were run on a Linux Intel Core i7, 3.40 $\mathrm{GHz}$ CPU with $8 \mathrm{~GB}$ of main memory. The benchmarks are taken from LGSynth93 [24]. Multi-output benchmarks were synthesized by minimizing each output independently from the others. To show the performance in area of the circuits derived by using Boolean relations, we generated SOP forms of $(u, v)$ using ESPRESSO both in exact and heuristic mode. To evaluate the obtained circuits, the SIS system was used with the MCNC technology library for mapping and the SIS command map $-\mathrm{W}-\mathrm{f} \quad 3-\mathrm{s}$ to estimate area and delay.

In Table IV we compare synthesis time (in seconds), mapped area and delay of circuits and SOP forms for a significant subset of the benchmarks. The first column reports the names of the benchmarks. The following columns report, by groups of three, the synthesis times in seconds, the areas and delays estimated by SIS. The first two groups, labeled SOP exact and SOP heuristic, refer to plain SOPs. The next group refers to circuits synthesized with different op gates ( $\notin$, $\Rightarrow$, and XNOR).

Table V summarizes the comparison between op circuits and SOP forms for both exact and heuristic minimization. In more detail, we report the percentages of benchmarks where we obtain better results with respect to the corresponding SOP forms. We observe that a high percentage of op circuits synthesized with Boolean relations turned out to be more compact and yielded lower delays than the corresponding SOP forms. This is true for both the exact and heuristic mode.

Table VI reports the average gains obtained with op circuits with respect to SOP forms for both exact and heuristic minimization. We observe that the synthesis of the $o p$ circuits yields positive gains for both area and delay, at the expense of more computation times. Note that $o p$ circuits with an XNOR op gate exhibit a lower gain w.r.t. the corresponding benchmarks with an AND or OR op gate. On average, we obtain higher gains in the case of exact minimization. In particular, the maximum percentage of area gain is $71 \%$ ( $\max 128)$ in the exact case and $67 \%$ (Z9sym) in the exhaustive case (in these two examples, the percentage is the same for all op gates).

In Table VII we compare against approaches presented in [9] and in [10]. In [9] the authors describe a three-level logic synthesis procedure based on a novel strategy for pairing cubes. They ran the experiments on a Sun Ultra 60 operating with two $360 \mathrm{MHz} \mathrm{CPU}$ and with $1024 \mathrm{MB}$ RAM main storage. In [10] the authors describe a three-level heuristic AND-OR-XOR minimization strategy for incompletely specified Boolean functions. They ran their experiments on a Sun SPARC 20 operating at $50 \mathrm{MHz}$ with $64 \mathrm{MB}$ RAM main storage. The first and the second columns of the table report the names of the benchmarks and the number of inputs/outputs, respectively. The following six columns report both execution time (in seconds) and number of literals of minimized $o p$ circuits for each case ( $\nLeftarrow, \Rightarrow$, and XNOR) and two different cost functions: the first (SIS) minimizes the number of literals, and the second (BDD) minimizes the size of the BDDs used for representing the relations. Finally, the last columns report results presented in [9] (columns 15 and 16) and in [10] (columns 17 and 18). The results show that the op circuits synthesized with Boolean relations turned out to be more compact than the corresponding circuits proposed in [9] and in [10] in about $29 \%$ of our experiments. Finally, we compare computation time, area, delay and number of literals of $o p$ circuits with respect to the results of ESPRESSO when run 
with output phase assignment (to choose the best realization between the positive and negative phase of each output). After choosing a phase assignment for each output, the function is minimized (in heuristic or exact mode). In about $15 \%$ of tested benchmarks, we stopped the computation of ESPRESSO with output phase assignment (after about 30 minutes), without obtaining minimization results.

In Tab.VIII we compare synthesis time (in seconds), mapped area and delay of circuits synthesized with ESPRESSO after the phase optimization (ESPRESSO command-Dopo) against op circuits. The first column reports the names of the benchmarks. The following columns report, by groups of three, the synthesis time in seconds, the area and delay estimated by SIS. The first two groups refer to ESPRESSO synthesis. The next group refers to $o p$ circuits synthesized with different $o p$ gates $(\nLeftarrow, \Rightarrow$, and XNOR). In the exact case, the percentages of op circuits with lower computation time, area and delay w.r.t. the corresponding circuits minimized with ESPRESSO are $5 \%, 94 \%$, and $89 \%$, respectively. In the heuristic case, the percentages of op circuits with lower time, area and delay w.r.t. the corresponding circuits minimized with ESPRESSO are $87 \%, 75 \%$, and $77 \%$, respectively.

\section{CONCLUSIONS AND FUTURE WORK}

We considered the bi-decomposition of ISFs, which have the form $u$ op $v$, where $o p$ can be any two-input logic function. Then we characterized all the correct implementations in such a form in terms of logic functions compatible with a Boolean relation, depending on the operator op. We studied the taxonomy of such circuits, and classified them into three groups according to the chosen op gate. Any member of a group can be transformed into any other member of the same group by complementing one or more of the inputs. Then we experimented with one example op from each group, namely op $\in\{\nLeftarrow, \Rightarrow$, and XNOR $\}$. These were chosen to differ from other forms already studied in the literature. Finally, we reported experiments to compare such realizations vs. SOPs as well as other published three-level forms, in term of area and delay, evaluated by synthesizing and mapping the circuits with SIS. This showed good gains in a majority of benchmarks against affordable increases in synthesis runtime.

Future work includes completing an exhaustive study of all ten non-trivial ops, and in finding a way to choose the best $o p$ for a particular ISF. This might be based on solving a phase assignment problem within each of the three group classifications. A variant of BREL might be developed to examine, at each of its steps, choosing a function or its complement implementation. Moreover, since in general we have multi-output ISFs to implement, treating them all at once using a single Boolean relation would be of great interest. Another interesting direction would be to iterate the construction of the blocks $u$ and $v$ recursively to obtain multi-level bidecompositions with higher depths. Also, inclusion of a MUX as an $o p$ would be interesting. The question of taking better advantage of logic sharing among the $2 m$ outputs is another potential direction of investigation.

\section{REFERENCES}

[1] D. Bañeres, J. Cortadella, and M. Kishinevsky, "A Recursive Paradigm to Solve Boolean Relations," IEEE Transactions on Computers, vol. 58, no. 4, pp. 512-527, April 2009.
[2] A. Bernasconi, V. Ciriani, R. Drechsler, and T. Villa, "Logic Minimization and Testability of 2-SPP Networks," Computer-Aided Design of Integrated Circuits and Systems, IEEE Transactions on, vol. 27, no. 7, pp. 1190-1202, July 2008.

[3] R. Brayton and F. Somenzi, "An Exact Minimizer for Boolean Relations," in Computer-Aided Design, 1989. ICCAD-89. Digest of Technical Papers., 1989 IEEE International Conference on, 1989, pp. 316-319.

[4] V. Ciriani, "Synthesis of SPP Three-Level Logic Networks using Affine Spaces," IEEE Trans. on CAD of Integrated Circuits and Systems, vol. 22, no. 10, pp. 1310-1323, 2003.

[5] V. Ciriani and A. Bernasconi, "2-SPP: a Practical Trade-Off between SP and SPP Synthesis," in 5th International Workshop on Boolean Problems (IWSBP2002), 2002, pp. 133-140.

[6] D. Debnath and T. Sasao, "Minimization of AND-OR-EXOR ThreeLevel Networks with AND Gate Sharing," IEICE Trans. Information and Systems, vol. E80-D, no. 10, pp. 1001-1008, 1997.

[7] _ - "Multiple-Valued Minimization to Optimize PLAs with Output EXOR Gates," in IEEE International Symposium on Multiple-Valued Logic, 1999, pp. 99-104.

[8] D. Debnath and Z. Vranesic, "A Fast Algorithm for OR-AND-OR Synthesis," IEEE Transactions on CAD, vol. 22, no. 9, pp. 1166-1176, 2003.

[9] E. Dubrova and P. Ellervee, "A Fast Algorithm for Three-Level Logic Optimization," in Int. Workshop on Logic Synthesis, 1999, pp. 251-254.

[10] E. Dubrova, D. Miller, and J. Muzio, "AOXMIN: A Three-Level Heuristic AND-OR-XOR Minimizer for Boolean Functions," in 3rd International Workshop on the Applications of the Reed-Muller Expansion in Circuit Design, 1997, pp. 209-218.

[11] R.-R. Lee, J.-H. R. Jiang, and W.-L. Hung, "Bi-decomposing large boolean functions via interpolation and satisfiability solving," in Design Automation Conference, 2008. DAC 2008. 45th ACM/IEEE. IEEE, 2008, pp. 636-641.

[12] F. Luccio and L. Pagli, "On a New Boolean Function with Applications," IEEE Transactions on Computers, vol. 48, no. 3, pp. 296-310, 1999.

[13] A. Malik, D. Harrison, and R. Brayton, "Three-Level Decomposition with Application to PLDs," in IEEE International Conference on Computer Design: VLSI in Computer \& Processors, ICCD '91, 1991, pp. 628-633.

[14] A. Mishchenko, B. Steinbach, and M. Perkowski, "An algorithm for bidecomposition of logic functions," in Design Automation Conference, 2001. Proceedings, 2001, pp. 103-108.

[15] A. Mishchenko, R. Brayton, and S. Chatterjee, "Boolean factoring and decomposition of logic networks," in Proceedings of the 2008 IEEE/ACM International Conference on Computer-Aided Design. IEEE Press, 2008, pp. 38-44.

[16] M. Perkowski and S. Grygiel, "A Survey of Literature on Function Decomposition," PSU Electrical Engineering Department, Technical Report November 20, 1995, 1995.

[17] R. Rudell and A. Sangiovanni-Vincentelli, "Multiple Valued Minimization for PLA Optimization," IEEE Transactions on CAD, vol. 6, no. 5, pp. 727-750, 1987.

[18] T. Sasao, "Input Variable Assignment and Output Phase Optimization of PLA's," Computers, IEEE Transactions on, vol. C-33, no. 10, pp. 879-894, Oct 1984.

[19] — "On the Complexity of Three-Level Logic Circuits," in Int. Workshop on Logic Synthesis, 1989.

[20] — , "OR-AND-OR Three-Level Networks," in Representation of Discrete Functions, T. Sasao and M. Fujita, Eds. Kluwier Academic, 1996.

[21] T. Sasao and J. Butler, "On bi-decomposition of logic functions," in Int. Workshop on Logic Synthesis, 1997.

[22] C. Scholl, Functional Decomposition with Applications to FPGA Synthesis. Springer, 2002.

[23] C. Yang and M. Ciesielski, "Bds: a bdd-based logic optimization system," Computer-Aided Design of Integrated Circuits and Systems, IEEE Transactions on, vol. 21, no. 7, pp. 866-876, 2002.

[24] S. Yang, "Logic synthesis and optimization benchmarks user guide version 3.0," Microelectronic Center, User Guide, 1991. 